\title{
Causal effect of prolificacy on milk yield in dairy sheep using propensity score
}

\author{
Vera C. Ferreira, ${ }^{*}$ David L. Thomas, ${ }^{*}$ Bruno D. Valente, ${ }^{*} \dagger$ and Guilherme J. M. Rosa ${ }^{*} \ddagger^{1}$ \\ *Department of Animal Sciences, \\ †Department of Dairy Science, and \\ ‡Department of Biostatistics and Medical Informatics, University of Wisconsin, Madison 53706
}

\section{ABSTRACT}

In animal production, it is often important to investigate causal relationships among variables. The gold standard tool for such investigation is randomized experiments. However, randomized experiments may not always be feasible, possible, or cost effective or reflect real-world farm conditions. Sometimes it is necessary to infer effects from farm-recorded data. Inferring causal effects between variables from field data is challenging because the association between them may arise not only from the effect of one on another but also from confounding background factors. Propensity score (PS) methods address this issue by correcting for confounding in different levels of the causal variable, which allows unbiased inference of causal effects. Here the objective was to estimate the causal effect of prolificacy on milk yield (MY) in dairy sheep using PS based on matched samples. Data consisted of 4,319 records from 1,534 crossbred ewes. Confounders were lactation number (first, second, and third through sixth) and dairy breed composition $(<0.5,0.5-0.75$, and $>0.75$ of East Friesian or Lacaune). The causal variable prolificacy was considered as 2 levels (single or multiple lambs at birth). The outcome MY represented the volume of milk produced in the whole lactation. Pairs of single- and multiple-birth ewes $(1,166)$ with similar PS were formed. The matching process diminished major discrepancies in the distribution of prolificacy for each confounder variable indicating bias reduction (cutoff standardized bias $=20 \%$ ). The causal effect was estimated as the average difference within pairs. The effect of prolificacy on MY per lactation was $20.52 \mathrm{~L}$ of milk with a simple matching estimator and $12.62 \mathrm{~L}$ after correcting for remaining biases. A core advantage of causal over probabilistic approaches is that they allow inference of how variables would react as a result of

Received March 21, 2017.

Accepted May 30, 2017.

${ }^{1}$ Corresponding author: grosa@wisc.edu external interventions (e.g., changes in the production system). Therefore, results imply that management and decision-making practices increasing prolificacy would positively affect MY, which is important knowledge at the farm level. Farm-recorded data can be a valuable source of information given its low cost, and it reflects real-world herd conditions. In this context, PS methods can be extremely useful as an inference tool for investigating causal effects. In addition, PS analysis can be implemented as a preliminary evaluation or a hypothesis generator for future randomized trials (if the trait analyzed allows randomization).

Key words: causality, milk, prolificacy, propensity score

\section{INTRODUCTION}

In livestock research there are many scenarios in which the objective is to learn about causal relationships, such as the effect of diet and management practices on animal performance. Undertaking this task with observational studies is challenging because the observed association between a causal variable and an outcome not only stems from effects of the former on the latter but could also be from background confounding factors (Pearl, 2009). In other words, the probability of an individual being in each level of the causal variable is influenced by background factors that also affect the outcome variable, creating associations between the 2 variables that do not reflect causal effects.

An alternative for dealing with potential confounders is to use randomized experiments (Fisher, 1926). Randomized assignment of individuals to levels of the causal variable removes such confounding, leading to unbiased estimation of causal effects. For this reason, randomized experiments (Fisher, 1926) are considered the gold standard for causal learning. This process imposes equal probability of causal variable levels for each individual regardless of background confounding factors. This provides an interpretation of the difference in the average outcomes (in different categories of the causal variable), which is representative of the 
target causal effect. However, those experiments may not always be feasible, ethical, or economically viable (Rosenbaum, 2010).

In such cases, an alternative consists of analytical methods that investigate causality from observational data. Those tools for causal learning can be extremely useful in animal sciences because farmers routinely collect and store field data, making available large data sets with phenotypic, environmental, and management variables. Furthermore, farm-recorded data provide a better representation of field (real-world) conditions than data from experimental settings (Rosa and Valente, 2013). However, as mentioned previously, exploring observational data requires correcting for the influence of confounding factors, which can be achieved using appropriate methods, such as propensity score (PS).

The PS methodology developed by Rosenbaum and Rubin (1983) is a relatively new and innovative statistical tool for unbiased estimation of causal effects using nonexperimental data. It involves studying how background factors affect the probability of assignment of observational units to each level of the causal variable (traditionally categorical and named "treatment" in the PS literature) and correcting for imbalances. Propensity score analysis has been used in a variety of disciplines such as economics (e.g., Jordà and Taylor, 2016), epidemiology (e.g., Shi et al., 2016), and social sciences (e.g., Santín and Sicilia, 2016). This demonstrates how useful the method can be in different areas, but to our knowledge it has never been applied in the livestock context before.

Here we investigate the causal effect of prolificacy on milk yield (MY) in dairy sheep. It is likely that a direct comparison of MY between single- and multiple-birth ewes from field data is biased due to confounding factors such as breed composition and lactation number, as both are known to affect MY and prolificacy (Ruiz et al., 2000; Snowder et al., 2001). Previous randomized experiments in dairy sheep found a positive association between the number of corpora lutea (McKusick et al., 2002) as well as hormones involved with reproduction (Labussière et al., 1996) and MY. Nonetheless, those studies did not directly evaluate the effect of prolificacy. The relationship between prolificacy and MY has been investigated with field data in purebred (Cappio-Borlino et al., 1995; Ruiz et al., 2000) and crossbred (Gibb and Treacher, 1982) sheep in the context of quantitative genetic studies. These field data studies found a positive association between prolificacy and MY but did not apply any causal inference methods, and thus no causal conclusions were reached. The objective of this study was to use PS methods to estimate the causal effect of number of lambs born on MY in crossbred dairy sheep using information from farm-recorded data.

\section{MATERIALS AND METHODS}

\section{Data Description}

Data comprised 4,319 lactation records collected between 1997 and 2013 (with 1 lambing season/yr) from 1,534 crossbred dairy ewes from the Spooner Agricultural Research Station of the University of WisconsinMadison in northwest Wisconsin. The average breed composition consisted mostly of dairy breeds (East Friesian $=45.2 \%$; Lacaune $=27.6 \%$, with smaller percentages for Dorset (10.4\%), Polypay (5.0\%), Targhee $(3.2 \%)$, Romanov (2.6\%), Rideau (2.0\%), Commercial (1.1\%), Kathadin (1.0\%), Rambouillet (1.0\%), Finnsheep (0.8\%), Texel (0.1\%), and Hampshire (0.1\%). The Commercial breed category was assigned when breed composition was not known with certainty, but it most likely consisted of some combination of Targhee, Finnsheep, Romanov, and Dorset breeds.

Because the crossbred ewes comprised mainly the dairy breeds East Friesian and Lacaune, individual breed compositions were assigned into 2 explanatory covariates with 3 categories each: $<50 \%, 50$ to $75 \%$, and $>75 \%$ East Friesian or Lacaune. Lactation data were recorded from the first through sixth lactations. An ANOVA test indicated that the third through sixth lactations did not differ significantly $(P>0.05)$; therefore, they are considered here as a single category. Two levels were considered for the trait prolificacy, reflecting the number of lambs born per parturition as single or multiple (mostly twins, but triplets and quadruplets were also assigned to this category) births.

Lambs were left with their dams for 36 to $48 \mathrm{~h}$ after birth to ingest colostrum. After that period, lambs were artificially raised on ad libitum milk replacer, and ewes were machine milked twice a day (morning and afternoon) until test-day MY dropped below $0.5 \mathrm{~L}$. The trait MY corresponds to the total volume of milk produced per ewe for the whole lactation $($ mean $=268.5 \mathrm{~L}$, $\mathrm{SD}=116.4 \mathrm{~L}$ ). Only lactations longer than $80 \mathrm{~d}$ were included in the analysis.

\section{Statistical Analysis}

A set of steps is well stablished for performing PS analysis in other fields. For consistency, those 5 steps were also applied here. In step 1, the PS analysis requires declaring the confounder set of variables that could potentially affect both the causal variable (prolificacy) and the outcome (MY). This confounder set 
is often referred to as covariates in the PS literature. In step 2, the PS, which is defined as the conditional probability of assignment to a particular level of the causal variable given the values of the confounder set (Rosenbaum and Rubin, 1983), is computed for each observational unit (in this analysis, each lactation for a given ewe). The distribution of PS can be compared between levels of the causal variable to check whether systematic differences exist between levels. The presence of systematic differences is an indicator that confounding exists; therefore, corrections should be performed. In step 3, different PS techniques can be applied, such as matched samples (MS), which corrects for bias by comparing individuals with similar backgrounds. This involves forming pairs of individuals with different causal variable levels but similar PS values. After forming pairs, step 4 is a balance check, included in the analysis to make sure that no significant systematic differences in background factors remain between levels of the causal variable. Finally, in step 5, the causal effect can be estimated as the mean difference between outcome values within pairs. More details on each of these steps are provided next. The analysis, with all 5 steps, was conducted under a frequentist framework using the R package nonrandom (Stampf, 2014).

Step 1: Confounder Variables. The choice of the confounder set considered in this analysis (lactation number, East Friesian and Lacaune breed proportions) was made by testing different possible confounder variables combined with coherent biological reasoning. Many variables (e.g., year) were included in the model at first, and only those displaying simultaneous effect on both prolificacy and MY (i.e., causing confounding) were retained.

Step 2: Estimation of PS. The PS methods propose to infer the causal effect relying on a counterfactual framework (Neyman, 1923, 1935; Rubin, 1974; Holland, 1986). Ideally, inferring this effect involves comparing the outcome value of an individual in one level of the causal variable (e.g., single birth) with the outcome of the same individual under the other level of the causal variable (i.e., multiple birth). The problem with this comparison is that in practice only one instance is realized, and therefore the other outcome is always missing. The matched samples technique substitutes the missing potential outcome with the outcome of an ewe with similar background confounding effects (i.e., similar PS value) but under a different level of the causal variable (Guo and Fraser, 2010). Both small and large sample theory show that adjustment for the PS is sufficient to remove bias due to all observed confounders (Rosenbaum and Rubin, 1983).

In this analysis, the PS value for each ewe (i.e., the probability of single or multiple birth) was estimated as a function of the confounder set using the following logistic model:

$$
\eta_{i}=\beta_{0}+\beta_{1} X_{i 1}+\beta_{2} X_{i 2}+\beta_{3} X_{i 3}
$$

where $\eta_{i}$ is the linear predictor that maps to the binary trait (prolificacy) space of the $i$ th lactation record within an ewe through the logit function (with $i=1$, $\ldots, 4,319) ; \beta_{0}$ is an intercept; $X_{i k}$ (with $k=1,2,3$ ) are the confounding variables reflecting lactation order and the East Friesian and Lacaune breed proportions, respectively; and $\beta_{k}$ (with $k=1,2,3$ ) are the slopes for the above-described confounding variables, respectively.

Step 3: Matched Samples. An alternative for implementing the PS analysis proposed by Rosenbaum and Rubin (1983) is to use the matched samples technique for adjustment. This is the most widely applied PS method. As suggested by Lunt (2014), it is capable of producing matching as closely as possible and therefore is efficient in minimizing bias. For this reason, the matched samples approach was chosen here to calculate the causal effect.

After the calculation of the PS values, lactation records of single-birth ewes were paired up with those of multiple-birth ewes with similar PS levels. The criterion for similarity was defined by a caliper size (maximum distance between the PS values of each ewe) equal to $20 \%$ of 1 SD of the logit of the PS, as proposed by Austin and Mamdani (2006) and Austin (2009). This yielded a caliper size of 0.13 (link function scale) in this analysis. From all possible observation pairs within the caliper size, the one with the smallest PS difference was chosen first. This implies that matching will be as close as possible to reduce bias in the estimation of the causal effect but also implies that data points may be excluded from the analysis, reducing the sample size.

Step 4: Balance Diagnosis. It is important to check whether the matching pairs step was able to correct for confounding. More specifically, one can check whether matching on the PS led to similar distribution of prolificacy across different levels of each of the $k$ confounding variables, referred to as balance in the PS literature (Ho et al., 2007). Achieving balance indicates that the causal effect of prolificacy on MY can be estimated without bias. On the other hand, lack of balance indicates that the confounder set chosen may not be appropriate (Austin, 2009) and that other sets should be tested.

Balance can be assessed intuitively by visual inspection of graphs with the frequency distributions of each confounder variable (for each level of the causal variable) before and after matching. However, a more formal approach using statistical tools to compare the 
distributions is recommended. One alternative for statistically evaluating balance is studying standardized differences for each variable in the confounder set before and after matching. In this analysis, the standardized difference was defined as

$$
d_{k}=\frac{\left(\bar{x}_{m b, k}-\bar{x}_{s b, k}\right)}{\sqrt{\frac{s_{m b, k}^{2}+s_{s b, k}^{2}}{2}}}
$$

where $\bar{x}_{m b, k}$ and $\bar{x}_{s b, k}$ denote the sample mean of the $k$ th confounder variable in each prolificacy levels $(m b=$ multiple birth; $s b=$ single birth), and $s^{2}{ }_{m b, k}$ and $s^{2}{ }_{s b, k}$ denote the sample variance of the $k$ th confounder variable in each level of prolificacy.

Standardized differences were first applied for balance check in matched samples by Rosenbaum and Rubin (1985). This approach is appropriate because it corrects for the difference in means but also takes into account a measure of dispersion, being a good representation of the distribution (Austin and Mamdani, 2006; Austin, 2009). Although there is no clear consensus on the best threshold for standardized differences to be declared as null, in this analysis we decided to adopt a $20 \%$ threshold for standardized differences, as originally suggested by Rosenbaum and Rubin (1985).

Step 5: Estimation of Causal Effect. After matching pairs, the causal effect $(\tau)$ of prolificacy on MY can be inferred as

$$
\hat{\tau}=\frac{1}{n} \sum_{j=1}^{n}\left(y_{j}^{m b}-y_{j}^{s b}\right),
$$

where $j$ represents each of the $n$ matched pairs and $y$ is the MY of ewes with either single-birth $(s b)$ or multiple-birth $(m b)$ lambs. However, matching on the exact PS values is often impossible in practice. Abadie and Imbens (2002) show that if the matching is not exact, the estimate of the causal effect will still be biased in finite samples. More specifically, the magnitude of bias is proportional to the magnitude of the residual discrepancies on the PS within a pair. As the caliper size reduces, PS values inside the matched pairs tend to get more similar. However, although it leads to less bias, more information would tend to be wasted due to a greater number of individuals with a unique PS, which adversely affects the final number of matched pairs used and consequently the quality of inferences. In other words, there is a bias-variance trade-off; although reducing the caliper reduces bias, it tends to increase variance.
An alternative for dealing with such an issue is to estimate the causal effect by using the so-called biascorrected matching estimator (Abadie and Imbens, 2002; Guo and Fraser, 2010). This approach uses least squares regression to adjust for any remaining differences in the PS value within a matched pair. The correction is performed by modifying the value of one member in the pair as a function of the $E\left(Y_{t} \mid x_{k}\right)$, in which $Y_{t}$ is the outcome given the causal variable $t$ and $x_{k}$ are the confounding variables. It is important to realize that if residual imbalances are nonexistent, the bias-corrected estimator and the simple matching estimator should yield virtually identical values. This correction is especially needed when confounder variables are continuous, implying that exact matching is seldom possible. Here we calculate both the simple and biased-corrected estimators and compare their results.

Last, fitting a traditional linear regression does not provide, per se, causal learning (Pearl, 2009). However, for the sake of comparison of results with traditional techniques, estimates from both marginal and multiple linear regressions of MY on prolificacy were calculated as well.

\section{RESULTS AND DISCUSSION}

The distributions of estimated PS for each category of prolificacy are depicted in Figure 1. If there is no confounding, the estimated PS values should be virtually the same for individuals in both levels of the causal variable (i.e., the 2 distributions would completely overlap). However, we observe instead only partially overlapping distributions. These results suggest differences in the background factors of ewes in the 2 groups before lambing, indicating the possibility of confounding when inferring the causal effect of prolificacy on MY. In other words, inferring the causal effect from direct comparisons between average MY of the 2 categories of prolificacy might provide biased results (Rosenbaum, 2010).

A total of 1,166 pairs of single- and multiple-birth ewes with similar PS values were formed in the application of the matched samples method; therefore, $54 \%$ of the available observations were used to infer the causal effect (i.e., any observation without a match within the caliper size was not used). Table 1 presents MY descriptive statistics and compares the frequencies of each level of the confounder variables for both prolificacy levels before (4,319 lactation records) and after (2,332 lactation records) matching pairs. In the rows relative to lactation, East Friesian breed percentage, and Lacaune breed percentage, we noticed that matching pairs strongly decreased the frequency of discrepancies between levels. The balance of the background 


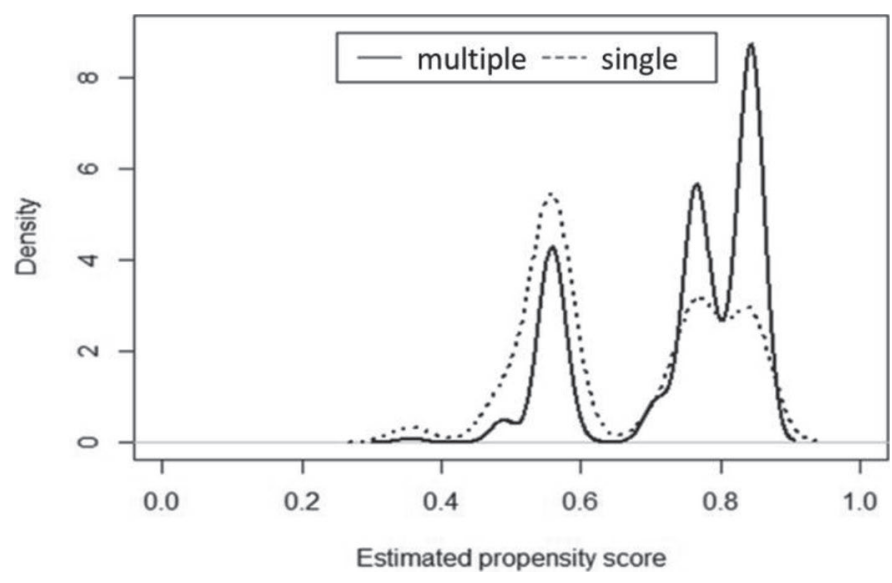

Figure 1. Distribution of estimated probabilities of assignment (propensity score) in each prolificacy level (single and multiple lamb birth) conditionally on the confounders (lactation number and breed composition of East Friesian and Lacaune).

covariates due to matching pairs is also represented in Figure 2. Statistical tests for checking balance are presented in Table 2. Absolute standardized differences before and after matching indicated that all variables were deemed balanced after matching (cutoff for the standardized bias $=20 \%$ ). The largest absolute value of standardized differences in the original data set was for the variable lactation $(61.9 \%)$. After matching, the same statistic was reduced to $16.9 \%$, which was below the cutoff point for standardized differences. Balance did not change much for the Lacaune (from $15.1 \%$ to $5.1 \%$ ) and East Friesian (from $4.5 \%$ to $8.5 \%$ ) breeds, which were still below the cutoff adopted in this analysis. Because the breed effects seem to be balanced before matching, a separate analysis was conducted excluding those variables from the confounder set. It was observed, however, that balance could not be achieved for lactation number when excluding those variables. For this reason, breed effects were maintained in the analysis.

Table 3 presents estimates of the PS matched samples causal effect of prolificacy on MY. The simple matching estimated effect was $20.52 \mathrm{~L}$ [SE $=3.77 \mathrm{~L}, 95 \%$ confidence interval $(\mathrm{CI})=13.13-27.91 \mathrm{~L}]$, whereas the biascorrected matching estimate was $12.62 \mathrm{~L}(\mathrm{SE}=3.63 \mathrm{~L}$, 95\% CI $=5.50-19.74)$. In both cases the CI does not include 0 , meaning that the difference in means of the single- and multiple-birth groups is significant $(P<$ 0.05). Under the assumptions of the methodology, such difference can be interpreted as an inferred causal effect. More specifically, and in the counterfactual sense, results suggest that an ewe that gave birth to a single lamb would be expected to have MY increased by 20.52 $\mathrm{L}$ (considering the simple match estimator) or $12.62 \mathrm{~L}$

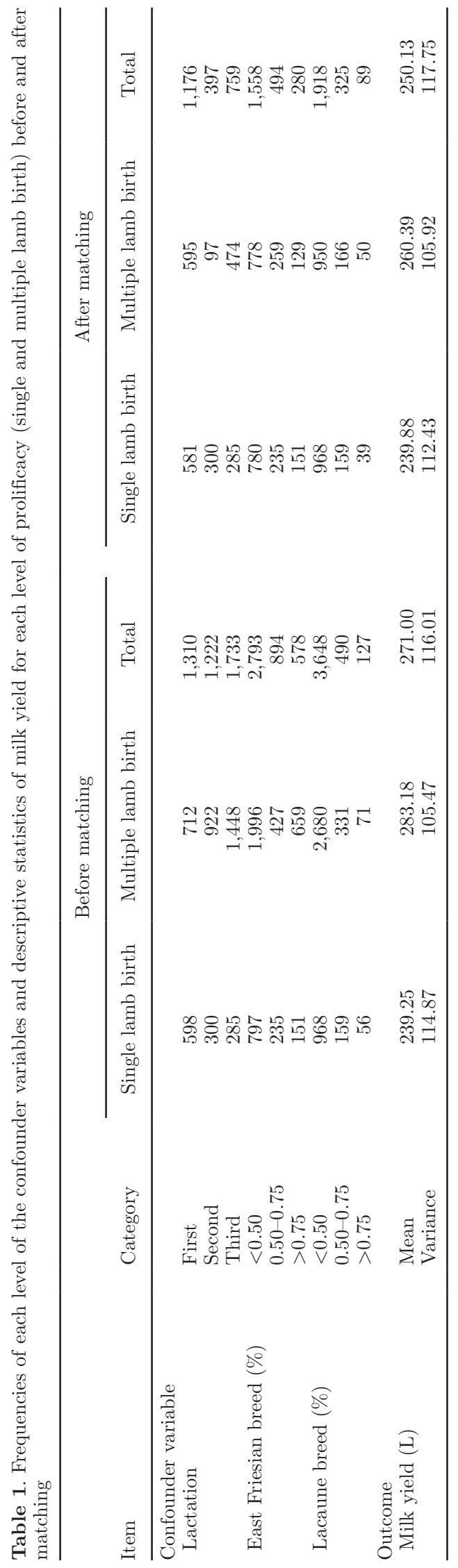

Journal of Dairy Science Vol. 100 No. 10, 2017 
Table 2. Standardized differences $(\%$; cutoff point $=20 \%)$ for each confounder before and after matching

\begin{tabular}{lcc}
\hline & \multicolumn{2}{c}{ Standardized difference } \\
\cline { 2 - 3 } Confounder variable & Before matching & After matching \\
\hline Lactation & 61.94 & $16.86^{1}$ \\
East Friesian breed proportion & $4.51^{1}$ & $8.54^{1}$ \\
Lacaune breed proportion & $15.12^{1}$ & $5.10^{1}$ \\
\hline
\end{tabular}

${ }^{1}$ Balanced according to the cutoff of $20 \%$.

(considering the bias-corrected estimator) if she had given birth to multiple lambs instead.

The values of the simple and biased corrected estimators were quite different. As indicated previously, it is expected that if no bias is left, these 2 estimators should yield equivalent values after matching. Therefore, one might interpret this difference as a suggestion of some remaining bias under the simple matching process. In this context, the bias-corrected causal effect $(12.62 \mathrm{~L})$ of prolificacy on MY might be a better representation of the true effect.

Both the logistic regression used to estimate the PS and the paired $t$-test used to estimate the significance of the causal effect (used to construct the CI) assume that observations and matched pairs, respectively, are independent. However, the data set used in this analysis contains repeated observations on the same individual as well as observations on individuals that are genetically related. Therefore, the independence assumption could be violated in both steps of analysis. Violating the independence assumption may inflate precision of estimates because the effective number of independent pairs (and their respective degrees of freedom) is in fact smaller than what it is assumed in the analysis.

The estimated positive causal effect of prolificacy on MY is in agreement with prior biological knowledge regarding the role of the placenta in MY. The placenta acts as an endocrine organ that actively participates in the development of the mammary glands in ruminants in the second half of pregnancy (Convey et al., 1973).

Table 3. Estimated causal effect of prolificacy on milk yield using propensity scores with matched samples as well as marginal and partial regression of milk yield on prolificacy

\begin{tabular}{lccc}
\hline Item & Effect & SE & 95\% CI \\
\hline Simple matching & $20.52^{*}$ & 3.77 & 13.13 to 27.91 \\
Bias-corrected matching & $12.62^{*}$ & 3.63 & 5.50 to 19.74 \\
Marginal regression $^{1}$ & $43.93^{*}$ & 3.87 & 36.34 to 51.52 \\
Partial linear regression $^{2}$ & 3.25 & 3.21 & -3.04 to 9.56 \\
\hline
\end{tabular}

${ }^{1}$ Milk yield as a function of prolificacy only.

${ }^{2}$ Milk yield as a function of prolificacy, lactation number, East Friesian proportion, and Lacaune proportion.

${ }^{*} P<0.05$.
Multiple-birth ewes tend to have more placental tissue than single-birth ewes. Increasing the volume of placental tissue might therefore improve mammary gland development, thus increasing MY. Endogenous mater-

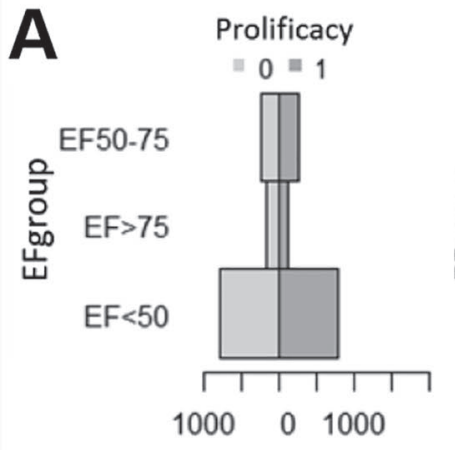

Number of observations

Matched Sample

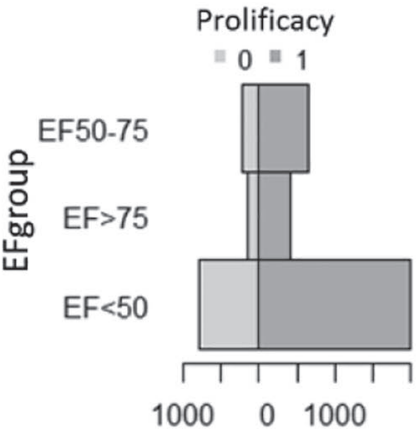

Number of observations

Original
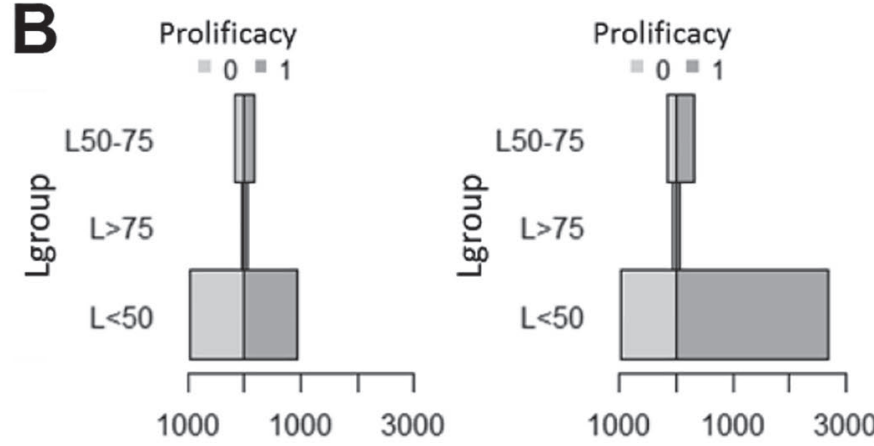

Number of observations

Number of observations

Matched Sample

Original
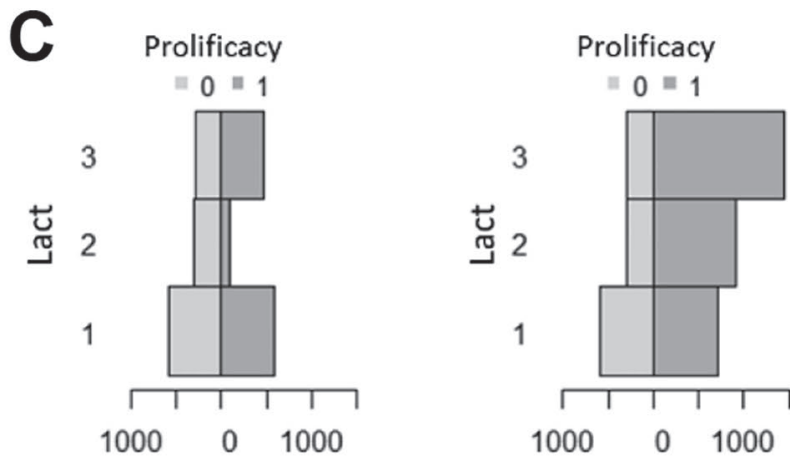

Number of observations

Number of observations

Matched Sample

Original

Figure 2. Distribution of prolificacy for each confounder variable (A: East Friesian breed composition, EFgroup; B: Lacaune breed composition, Lgroup; C: lactation, Lact) before and after matching. Prolificacy: 0 = single-birth ewes; 1 = multiple-birth ewes. 
nal hormones can also influence MY because they seem to be the primary stimulant of mammary development during the first half of pregnancy. They also play a role in milk production after birth in combination with suckling stimulus (Skjervold, 1977). However, in the present study all lambs were artificially raised on milk replacer after colostrum ingestion, so suckling should have very little effect on MY. For this reason, the effect of prolificacy on MY is likely even larger in systems in which lambs are raised by their dams.

Estimates for the marginal regression of MY on prolificacy are shown in Table 3. The results indicate that estimates are much larger in magnitude than the causal effect inferred with the PS approach. Marginal regression might be the starting point for domain researchers; however, it is important to understand how and why the estimates change as the model becomes more sophisticated. In this specific scenario, if there was no confounding, both the marginal regression and PS should yield virtually the same result. However, the fact that the results between them were so different indicates that the marginal regression may be suffering from bias, highlighting the importance of correcting for confounding.

On the other hand, for the multiple linear regression (results in Table 3), the estimated partial regression coefficient was of a much lower magnitude. It could be argued that the multiple regression fitted here could be used to infer the causal effect according to graph-based causal inference methods (Pearl, 2009). More specifically, fitting the multiple regression model identifies the effect of prolificacy on MY according to the so-called back-door criterion if the same set of confounders is assumed. One might therefore expect the inferred regression coefficient to resemble the PS-based estimated effect. However, that resemblance was not observed. One possible explanation for this difference is that the nature of the relationship between the confounders and MY is nonlinear (i.e., it could not be captured properly with a linear model). In such a scenario, PS may be advantageous compared with regression methods because the latter requires that departures from linearity between confounders and outcome be explicitly modeled. Propensity score is shown to be more robust than regression approaches for modeling misspecification regarding nonlinearity (Drake, 1993). Moreover, with an increased number of covariates, it becomes more challenging to properly model nonlinear effects, and PS methods may then be even more advantageous than multiple regression techniques.

The chief advantage of causal inference methods (as the PS approach) over probabilistic or predictive modeling is the inference of how variables, such as MY, would react as a result of external interventions on other vari- ables, such as prolificacy (Pearl, 2009). Therefore, these results reflect the effect of management practices that increase (decrease) prolificacy (e.g., treatment with progestin sponge, superovulation with FSH and LH, nutritional flushing before the mating season, or even selecting the flock for genes that are known to affect prolificacy) on MY. Those results cannot be supported by standard regression modeling unless it is applied within a causal inference framework (Pearl, 2009; Valente et al., 2015). As mentioned by Rosa and Valente (2013), many scenarios in animal production systems involve choosing management practices as a way of performing a positive intervention on production. These decisions depend on understanding the consequences of different sets of interventions, which in turn requires knowledge of causal effects between variables (Rosa and Valente, 2013). Practices that affect prolificacy can be considered as a frequent intervention in dairy ewes; therefore, understanding their consequences on the variables is important for predicting economic gains for the production system.

Another important aspect of this analysis is that breed effects were identified as confounders, indicating that genetic confounding might play an important role when inferring the effect between 2 heritable traits. However, this also indicates that even within purebreds there is probably relevant confounding due to genetic covariation. These factors were not taken into account here but potentially could be accounted for with the inclusion of genetic merit information of each animal in PS analysis of functional relationships between phenotypic traits. Even though correction for genetic confounding caused by linkage disequilibrium has been used in candidate gene studies (Zhao et al., 2009), to our knowledge this application for genetic confounding when investigating the causal effect between phenotypes has not been studied yet. This is certainly a topic that deserves further investigation in the context of PS and causal inference.

It is also important to stress that for traits such as prolificacy, it would be virtually impossible to randomize individuals to categories of single and multiple lambs born. Even if in vitro fertilization was applied in an experimental setting, it does not guarantee that the exact number of embryos implanted would develop to term. For traits for which randomization is not possible, the methodology presented here offers another valuable alternative for explicitly investigating causal relationships among variables from observational data in livestock. Nevertheless, often in livestock, the variable under study can be randomized. In this case, randomized control trials would be a more reliable tool for inferring causal effects. Still, in those scenarios, PS methods could be used as a preliminary evalua- 
tion or hypothesis generator for later validation using controlled trials.

At the same time, the implementation of experiments may sometimes involve changing the environmental conditions that animals are exposed to. This process itself may affect results such that they no longer reflect real-world conditions, as discussed by Rosa and Valente (2013). In those scenarios, results obtained from farm-recorded data may become an important source of information. It is also relevant to stress that data are already extensively collected at the farm level (Rosa and Valente, 2013). Therefore, a large amount of information may be already available for the analysis of functional relationships among phenotypes. In this scenario, PS methods emerge as a useful and easy-to-apply tool for investigating causal relationships at a much lower cost than implementing randomized experiments.

\section{ACKNOWLEDGMENTS}

This work was supported by grant WIS01815, "Exploring Causal Relationships Underlying Economically Important Traits in Dairy Sheep," from USDA (Washington, DC) to G. J. M. Rosa and D. L. Thomas.

\section{REFERENCES}

Abadie, A., and G. W. Imbens. 2002. Simple and bias-corrected matching estimators [Technical report]. Accessed Aug. 24, 2015. http://www.nber.org/papers/t0283.pdf.

Austin, P. C. 2009. Balance diagnostics for comparing the distribution of baseline covariates between treatment groups in propensityscore matched samples. Stat. Med. 28:3083-3107.

Austin, P. C., and M. M. Mamdani. 2006. A comparison of propensity score methods: A case-study estimating the effectiveness of postAMI statin use. Stat. Med. 25:2084-2106.

Cappio-Borlino, A., G. Pulina, and G. Rossi. 1995. A non-linear modification of Wood's equation fitted to lactation curves of Sardinian dairy ewes. Small Rumin. Res. 18:75-79.

Convey, E. M., J. W. Thomas, H. A. Tucker, and J. L. Gill. 1973. Effect of thyrotropin releasing hormone on yield and composition of bovine milk I. J. Dairy Sci. 56:484-486.

Drake, C. 1993. Effects of misspecification of the propensity score on estimators of treatment effect. Biometrics 49:1231-1236.

Fisher, R. A. 1926. The arrangement of field experiments. J. Ministry Agric. Gr. Brit. 33:503-513.

Gibb, M. J., and T. T. Treacher. 1982. The effect of body condition and nutrition during late pregnancy on the performance of grazing ewes during lactation. Anim. Prod. 34:123-129.

Guo, S., and M. W. Fraser. 2010. Propensity Score Analysis: Statistical Methods and Applications. Sage, Thousand Oaks, CA.

Ho, D. E., K. Imai, G. King, and E. A. Stuart. 2007. Matching as nonparametric preprocessing for reducing model dependence in parametric causal inference. Polit. Anal. 15:199-236.
Holland, P. W. 1986. Statistics and causal inference. J. Am. Stat. Assoc. $81: 945-970$.

Jordà, Ò., and A. M. Taylor. 2016. The time for austerity: Estimating the average treatment effect of fiscal policy. Econ. J. (Oxf.) 126:219-255.

Labussière, J., P. G. Matnet, F. A. de la Chevalerie, and J. F. Combaud. 1996. Répétition de traitements progestatifs (FGA) et gonadostimulants (FSH et LH) pendant la phase descendante de la lactation de brebis Lacaune. Effets sur la production et la composition du lait et sur sa répartition alvéolaire et citernale. Ann. Zootech. 45:159-172.

Lunt, M. 2014. Selecting an appropriate caliper can be essential for achieving good balance with propensity score matching. Am. J. Epidemiol. 179:226-235.

McKusick, B. C., M. C. Wiltbank, R. Sartori, P. G. Marnet, and D. L. Thomas. 2002. Effect of presence or absence of corpora lutea on milk production in East Friesian dairy ewes. J. Dairy Sci 85:790-796.

Neyman, J. 1923. On the application of probability theory to agricultural experiments. Essay on principles. Stat. Sci. 5:465-472.

Neyman, J. 1935. Statistical problems in agricultural experimentation. J. R. Stat. Soc. 2:107-180.

Pearl, J. 2009. Causality. 2nd ed. Cambridge University Press, Cambridge, United Kingdom.

Rosa, G. J. M., and B. D. Valente. 2013. Breeding and genetics symposium: Inferring causal effects from observational data in livestock. J. Anim. Sci. 91:553-564.

Rosenbaum, P. R. 2010. Observational Studies. 2nd ed. Springer, New York, NY.

Rosenbaum, P. R., and D. B. Rubin. 1983. The central role of the propensity score in observational studies for causal effects. Biometrika 70:41-55.

Rosenbaum, P. R., and D. B. Rubin. 1985. Constructing a control group using multivariate matched sampling methods that incorporate the propensity score. Am. Stat. 39:33-38.

Rubin, D. B. 1974. Estimating causal effects of treatments in randomized and nonrandomized studies. J. Educ. Psychol. 66:688-701.

Ruiz, R., L. M. Oregui, and M. Herrero. 2000. Comparison of models for describing the lactation curve of latxa sheep and an analysis of factors affecting milk yield. J. Dairy Sci. 83:2709-2719.

Santín, D., and G. Sicilia. 2016. Does family structure affect children's academic outcomes? Evidence for Spain. Soc. Sci. J. 53:555-572.

Shi, J., B. Lu, K. Wheeler, and H. Xiang. 2016. Unmeasured confounding in observational studies with multiple treatment arms: Comparing mortality of severe trauma patients by trauma center level. Epidemiology 27:624-632.

Skjervold, H. 1977. The effect of foetal litter size on milk yield : Cross fostering experiments with mice. Z. Tierzucht. Zuchtbiol. 94:66-75.

Snowder, G. D., A. D. Knight, L. D. Van Vleck, C. M. Bromley, and T. R. Kellom. 2001. Usefulness of subjective ovine milk scores: I. Associations with range ewe characteristics and lamb production. J. Anim. Sci. 79:811-818.

Stampf, S. 2014. Nonrandom: Stratification and matching by the propensity score. R package version 1.42. Accessed Aug. 24, 2015. http://cran.r-project.org/web/packages/nonrandom/index.html.

Valente, B. D., G. Morota, F. Peñagaricano, D. Gianola, K. Weigel, and G. J. M. Rosa. 2015. The causal meaning of genomic predictors and how it affects construction and comparison of genomeenabled selection models. Genetics 200:483-494.

Zhao, H., T. R. Rebbeck, and N. Mitra. 2009. A propensity score approach to correction for bias due to population stratification using genetic and non-genetic factors. Genet. Epidemiol. 33:679-690. 